\title{
Performance Analysis of Softwarized Local Mobile Networks
}

\author{
Yushan Siriwardhana*, Pawani Porambage ${ }^{\dagger}$, Madhusanka Liyanage ${ }^{\ddagger}$, Mika Ylianttila ${ }^{\S}$ \\ ${ }^{*} \dagger \ddagger \S$ Centre for Wireless Communications, University of Oulu, Finland \\ ${ }^{\ddagger}$ School of Computer Science, University College Dublin, Ireland \\ Email: ${ }^{* \dagger \ddagger}$ [firstname.lastname]@oulu.fi, ${ }^{\ddagger}$ madhusanka@ ucd.ie
}

\begin{abstract}
The ever growing and revolutionizing demands in telecommunication industry to facilitate numerous business verticals are pushing towards more softwarized mobile communication technologies. Utilizing the capabilities of network softwarization, a novel telecommunication concept of local mobile network has been developed. The local mobile networks are getting popular due to their capability of providing efficient and reliable local services to a focused use case with higher flexibility. This paper presents the practical implementation aspects of a softwarized local mobile network and compare its performance with a conventional mobile network and a hybrid network.

Index Terms-5G, L5GO, SDN, NFV, Testbed
\end{abstract}

\section{INTRODUCTION}

Network Softwarization revolutionize the way network and computing infrastructures are designed and operated to deliver the services. Software-Defined Networking (SDN) and Network Function Virtualization (NFV) are the two pillars of network softwarization. SDN decouples the user and control planes enabling the programmability of the networks and NFV allows network functions to run as virtual instances on commodity hardware[1]. Softwarized networks are easily scalable to meet the increasing network demands, capable of sharing computing and storage resource to increase the efficiency of resource utilization.

The future wireless communication applications require service providers to put more emphasis on the case specific and location specific service delivery in different vertical sectors such as automotive, health, energy, industry and media. These requirements are expanding beyond the current capabilities of traditional Mobile Network Operators (MNO) whose services are often designed to serve masses. Hence, the need of locally deployed operators such as micro Operators $(\mathrm{uO})$ [2] to cater for such service requirements is evident. Local operators enable efficient and reliable local service deliveries with shorter deployment timelines due to its specific focus on the use case.

Two architectural options can be derived for softwarized mobile operator networks based on the serving use case, coverage area, the geographic distribution and ownership of the network components as illustrated in Figure 1.

1) Local Mobile Operator Architecture: A locally deployed network to provide services for specific use cases such as in a factory, hospital, university, shopping mall. The network includes locally deployed access network and the core network components as depicted in Figure 1a.
2) Hybrid Architecture: In a hybrid architecture, the network entities are not necessarily confined to one physical location, especially the core network. The network can be deployed with the collaboration of multiple operators providing different entities (i.e. core network elements, base stations), functioning together to provide service(s) required as shown in Figure 1b.

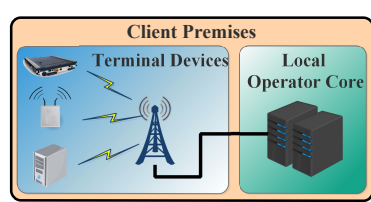

(a) Local Operator

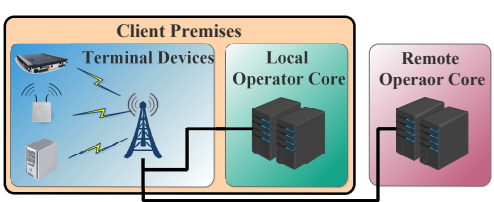

(b) Hybrid
Fig. 1: Architectural Options

The remainder of the paper is organized as follows: Section II explains the testbed implementation procedure and discusses the results. Finally, Section III concludes the paper.

\section{TEStBed Implementation AND Results}

The paper compares performance of three deployment options for mobile operator networks, i.e. pure local operator, hybrid model and the MNO.

\section{A. Testbed Environment}

1) Pure local operator: Testbed implementation of the pure local operator is done by integrating a $4 \mathrm{G}$ base station provided by Nokia, the EPC from Cumucore [3] and generic mobile devices. This setup was used as a local operator which has its own core network, access network and subscribers. The network diagram for the setup is illustrated in Figure 2 and Figure 3 depicts the actual setup of the local operator network.

2) Hybrid Model: As the hybrid network setup, we used (5GTN) [4] which is available in University of Oulu premises. Figure 4 illustrates the overall architecture of 5GTN. It is comprised of two interconnected environments located at University of Oulu (UO) and Nokia's Tamapare office. The site is UO contains one macro cell, few small cell eNodeBs (eNBs) and some of the core network functionalists such as Packet Data Network Gateway (PGW), Serving Gateway (SGW), Mobility Management Entity (MME). Other core network functionalities run from a remote core network, located at Nokia premises in Tampere, Finland. The remote core network is connected over a Virtual Private Network (VPN). 


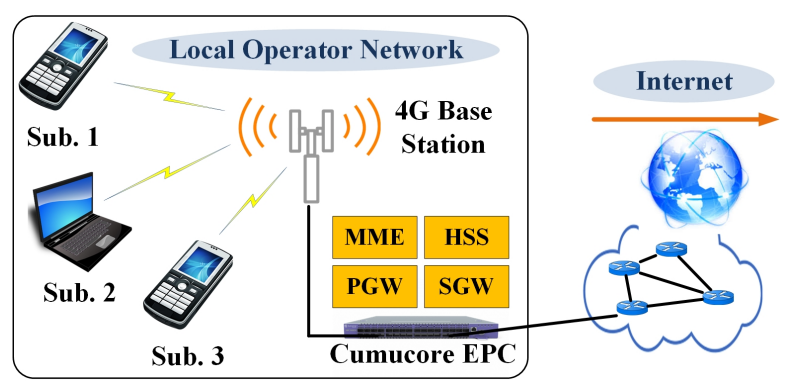

Fig. 2: Network Diagram of Experimental Setup

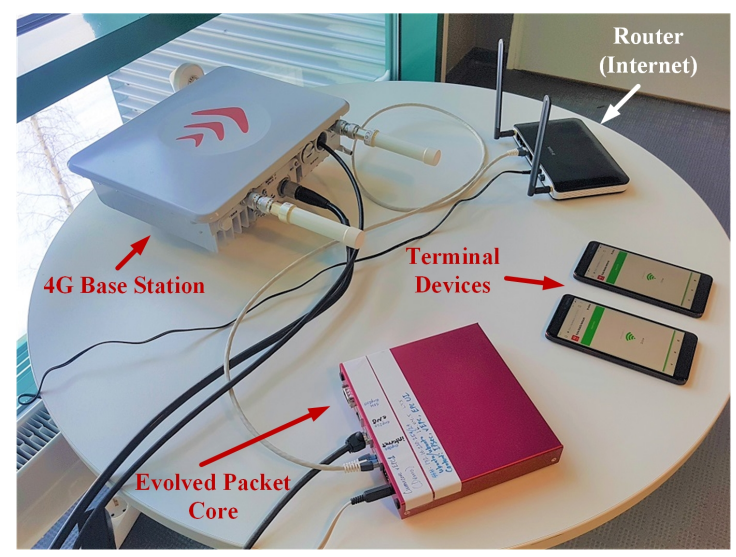

Fig. 3: Experimental Setup of the Devices

3) MNO: DNA SIM cards were used to implement MNO network. Based on 2019 financial statements, DNA positions as third service operator in Finland having 29\% market share in mobile communications and DNA's 4G network reaches almost $100 \%$ of the population in mainland Finland [5].

\section{B. Experiments}

We conduct two experiments under each network deployment model and obtain performance measurements for latency and throughput. Measurements for pure local operator and hybrid deployments were taken every two hours per day and an average of ten measurements were taken as the final value. For the MNO, experiment was conducted ten consecutive days and averaged to come up with a more realistic figure.

1) End-to-End Latency: We used "Network Analyzer" android application and used IPV6 ping command to measure the latency. Table I depicts the latency measurements with $95 \%$ confidence intervals. Latency is always the lowest for local operator network with less variations because of close proximity of the core network and less external traffic. Conversely, latency of MNO is higher with significant variations due to the core network distance and the variations in traffic congestion over time. For the hybrid network, latency is in between local operator values and MNO values and shows higher variation compared to the local operator.

2) Network Throughput: We used "Magic iPerf" android application and used IPV6 addresses for iperf command to measure the throughput. Table II depicts the throughput measurements with $95 \%$ confidence intervals. Observed

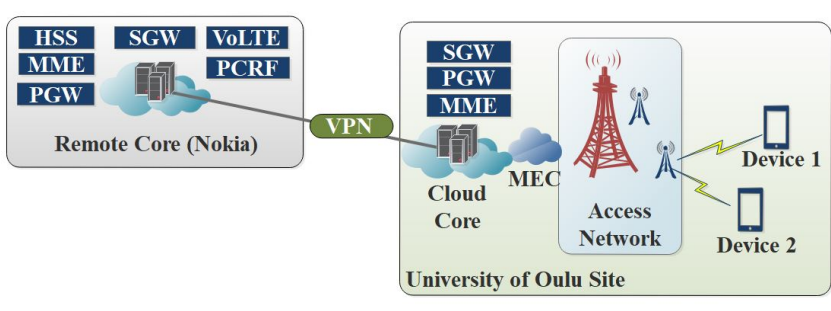

Fig. 4: Network Architecture of 5G Test Network [4]

TABLE I: Comparison of E2E latency

\begin{tabular}{|c|c|c|c|}
\hline Time of Day & Local (ms) & Hybrid (ms) & MNO (ms) \\
\hline 0800 & $78.63 \pm 3.52$ & $126.43 \pm 12.6$ & $245.49 \pm 27.2$ \\
1000 & $81.54 \pm 2.32$ & $125.09 \pm 11.2$ & $256.68 \pm 21.0$ \\
1200 & $80.41 \pm 2.33$ & $118.03 \pm 14.6$ & $102.74 \pm 14.1$ \\
1400 & $79.67 \pm 9.17$ & $122.23 \pm 17.6$ & $247.14 \pm 29.8$ \\
1600 & $82.39 \pm 7.56$ & $118.41 \pm 11.7$ & $212.34 \pm 23.1$ \\
1800 & $80.66 \pm 5.70$ & $115.35 \pm 13.7$ & $126.49 \pm 14.6$ \\
\hline
\end{tabular}

throughput is always higher for local operator and hybrid deployments compared to MNO. This is because the local and hybrid operators have less traffic originated from test devices compared to MNO, leading to less congestion. Conversely, throughput of MNO is lowest in all the cases. There could be multiple reasons for this. Traffic congestion at MNO network, operator policies such as QoS limits.

TABLE II: Comparison of throughput

\begin{tabular}{|c|c|c|c|}
\hline Time of Day & Local (Mbps) & Hybrid (Mbps) & MNO (Mbps) \\
\hline 0800 & $15.68 \pm 0.68$ & $15.83 \pm 1.20$ & $8.60 \pm 1.28$ \\
1000 & $17.10 \pm 1.23$ & $16.87 \pm 1.42$ & $6.53 \pm 1.37$ \\
1200 & $13.08 \pm 1.71$ & $15.91 \pm 1.02$ & $9.13 \pm 1.35$ \\
1400 & $13.87 \pm 2.10$ & $14.99 \pm 1.61$ & $4.20 \pm 1.78$ \\
1600 & $13.78 \pm 1.14$ & $14.36 \pm 2.25$ & $5.51 \pm 1.02$ \\
1800 & $14.26 \pm 0.97$ & $12.83 \pm 2.01$ & $6.52 \pm 1.45$ \\
\hline
\end{tabular}

\section{CONCLUSIONS}

This paper explores the benefits of utilizing local mobile operator networks and easily customize networks to serve specific use cases over the service provision via MNO. The paper presents the latency and throughput measurements for three deployment models called local operator, hybrid operator and MNO and argues that the selection of local and hybrid operators is beneficial in terms of performance measurements.

\section{ACKNOWLEDGEMENT}

This work is supported by Academy of Finland in 6G Flagship (\#318927) and 5GEAR (\#319669) projects.

\section{REFERENCES}

[1] M. Liyanage, A. Gurtov, and M. Ylianttila, Software Defined Mobile Networks (SDMN): Beyond LTE Network Architecture. John Wiley \& Sons, 2015.

[2] Y. Siriwardhana, P. Porambage, M. Ylianttila, and M. Liyanage, "Performance Analysis of Local 5G Operator Architectures for Industrial Internet," IEEE Internet of Things Journal, 2020.

[3] "Cumucore EPC." [Online]. Available: https://www.cumucore.com/

[4] "5G Test Network." [Online]. Available: https://www.5gtn.fi/

[5] “DNA Annual Report 2018." [Online]. Available: https://corporate.dna. fi/investors/reports/financial-reports 